\title{
Existence and Global Behavior of Decaying Solutions of a Nonlinear Elliptic Equation
}

\author{
Arij Bouzelmate, Mohamed El Hathout \\ Department of Mathematics \\ Faculty of Sciences \\ University Abdelmalek Essaadi \\ B.P. 2121, Tetouan \\ MOROCCO
}

Abstract: - This paper is devoted to study the following radial equation

$$
\left(\left|u^{\prime}\right|^{p-2} u^{\prime}\right)^{\prime}+\frac{N-1}{r}\left|u^{\prime}\right|^{p-2} u^{\prime}+\alpha|u|^{q-1} u+\beta r\left(|u|^{q-1} u\right)^{\prime}=0, \quad r>0 .
$$

where $p>2, q>1, N \geq 1, \alpha>0$ and $\beta>0$.

Our purpose is to give existence results of decaying solutions of the above equation and their asymptotic behavior near infinity. The study depends strongly of the sign of $N \beta-\alpha$ and the comparison between $\frac{\alpha}{q \beta}, \frac{p}{q+1-p}$ and $\frac{N-p}{p-1}$. More precisely, we prove that if $N \beta-\alpha>0$, there is a positive solution $u$ which has one of the following behaviors near infinity:

(i) $u(r) \underset{+\infty}{\sim} L r^{-\frac{\alpha}{q \beta}}$, where $L>0$

(ii) $u(r) \underset{+\infty}{\sim}\left(\left(\frac{p-1}{q \beta}\right)(q+1-p)\left(\frac{N-p}{p-1}-\frac{\alpha}{q \beta}\right)\left(\frac{\alpha}{q \beta}\right)^{p-1}\right)^{\frac{1}{q+1-p}} r^{-\frac{\alpha}{q \beta}}(\ln r)^{\frac{1}{q+1-p}}$

$($ iii $) u(r) \underset{+\infty}{\sim}\left(\frac{(p-1)\left(\frac{p}{q+1-p}\right)^{p-1}\left(\frac{N-p}{p-1}-\frac{p}{q+1-p}\right)}{\alpha-q \beta \frac{p}{q+1-p}}\right)^{\frac{1}{q+1-p}} r^{\frac{-p}{q+1-p}}$.

Key-Words: - Porous medium equation; fast diffusion equation; radial self-similar solutions; shooting method; decaying solutions; energy function.

Received: June 20, 2020. Revised: November 19, 2020. Accepted: December 3, 2020. Published: December 30, 2020. 


\section{Introduction and Main Results}

The aim of this paper is to investigate the structure of positive radial solutions to

$$
\Delta_{p} u+\alpha|u|^{q-1} u+\beta x . \nabla\left(|u|^{q-1} u\right)=0, \quad x \in \mathbb{R}^{N},
$$

where $p>2, q>1, N \geq 1, \alpha>0$ and $\beta>0$. As usual $\Delta_{p} u=\operatorname{div}\left(|\nabla u|^{p-2} \nabla u\right)$ is the $p-$ laplacien operator.

The idea of this work comes from the study of radial self-similar solutions to the following parabolic equation

$$
v_{t}=\Delta_{p} v^{m} \quad \text { in } \quad \mathbb{R}^{N} \times(0,+\infty),
$$

where $p>2$ and $0<m<1$.

When $p=2$, this equation becomes the porous medium equation, it appears in many physical models and it has been treated extensively in the literature, see [25] and [26]. When $m>1$, it is the slow diffusion equation, when $0<m<1$ it is the fast diffusion equation. When $m=1$, equation (2) reduce to the heat equation. See for example works of [1], [2], [3], [4], [7], [8], [9], [14], [15] and [21].

The radial self-similar solution to the parabolic equation (2) are the form

$$
v(x, t)=t^{-\alpha} W\left(t^{-\beta}|x|\right) .
$$

Where

$$
\alpha=\frac{\beta p-1}{1-m(p-1)}
$$

and $W$ is a radial positive solution which satisfies

$$
\Delta_{p} W^{m}(r)+\alpha W(r)+\beta r W^{\prime}(r)=0, \quad r>0 .
$$

For simplicity in the notation, we set $u=W^{m}$ and $q=\frac{1}{m}$ in (5), we obtain

$$
\Delta_{p} u+\alpha u^{q}+\beta r\left(u^{q}\right)^{\prime}=0, \quad r>0 .
$$

The question of the existence of a self-similar of equation (2) arises. We will prove that (2) admits a radial positive self-similar solution $v$ if $\frac{\alpha}{\beta}<N$ and $\frac{\alpha}{q \beta}<\frac{p}{q+1-p}$.

To obtain this result, we carry out a careful analysis of radial solutions of equation (11). Many authors have studied equation (11). If $p=2, \alpha=1$ and $\beta=0$, the first study is due to Emden-Fowler, see for example [10], [11] and [12]). He proved the existence results and give a classification of entire radial solutions. In the case $p=2, \alpha>0$ and $\beta>0$, equation (11) was studied by [18], [19], and [20]. When $p>2, \alpha=1$ and $\beta=0$, the first results are due to Ni and Serrin
[23]. Guedda and Veron [16] studied the existence of entire solutions in radial case. The non radial case was investigated by Bidaut- Veron and Pohozaev [5]. When $p>1, \alpha>0$ and $\beta=1$, equation (11) was studied by [22]. In the present work, we are interested in radial solutions of equation (1), we will study the following initial value problem.

Problem (P): Find a function $u$ defined on $[0,+\infty[$ such that $\left|u^{\prime}\right|^{p-2} u^{\prime}$ is in $C^{1}([0,+\infty[)$ and

$$
\left(\left|u^{\prime}\right|^{p-2} u^{\prime}\right)^{\prime}+\frac{N-1}{r}\left|u^{\prime}\right|^{p-2} u^{\prime}+\alpha|u|^{q-1} u+\beta r\left(|u|^{q-1} u\right)^{\prime}=0,
$$

$$
u(0)=a>0, \quad u^{\prime}(0)=0 .
$$

By reducing the problem $(P)$ to a fixed point for a suitable integral operator see (for example [6]), we prove that for each $a>0$, the problem $(P)$ has a unique global solution $u(., a, \alpha, \beta)$.

We focus our study to the case $N \beta-\alpha \geq 0$. If $N \beta-$ $\alpha=0$ and $q \geq p-1$, we find explicit solution of problem $(P)$

$$
u(r, a)= \begin{cases}a e^{\frac{-(p-1)}{p} \beta^{\frac{1}{p-1}} r^{\frac{p}{p-1}}} & \text { if } q=p-1 \\ \left(a^{\frac{p-1-q}{p-1}}+\frac{q+1-p}{p} \beta^{\frac{1}{p-1}} r^{\frac{p}{p-1}}\right)^{\frac{-(p-1)}{q+1-p}} & \text { if } q>p-1 .\end{cases}
$$

If $N \beta-\alpha=0$ and $q<p-1$, the solution $u(r, a)$ has compact support.

If $N \beta-\alpha>0$, we prove that $u(r, a)$ is a decaying solution for each $a>0$, i.e it is strictly positive and strictly decreasing on $(0,+\infty)$.

We are interested also to give asymptotic behavior of decaying solutions of problem $(P)$. For this purpose, let us represent equation (7) as an equivalent form.

For any real $c$, we set

$$
v_{c}(t)=r^{c} u(r) \text { where } r>0 \text { and } t=\ln (r) \text {. }
$$

Then, $v_{c}$ satisfies

$w_{c}^{\prime}(t)+A_{c} w_{c}(t)+\alpha e^{K_{c} t}\left|v_{c}\right|^{q-1} v_{c}(t)+q \beta e^{K_{c} t}\left|v_{c}\right|^{q-1} h_{c}(t)=0$

where

$$
\begin{gathered}
w_{c}(t)=\left|h_{c}\right|^{p-2} h_{c}(t) \\
h_{c}(t)=v_{c}^{\prime}(t)-c v_{c}(t)=r^{c+1} u^{\prime}(r) \\
A_{c}=N-p-c(p-1) \text { and } K_{c}=c(p-1-q)+p .
\end{gathered}
$$

We remark that three critical values of the parameter $c$ will be involved, $\frac{\alpha}{q \beta}, \frac{N-p}{p-1}$ and $\frac{p}{q+1-p}$. These values play an important role in the study of asymptotic behavior of positive solution of problem $(P)$. The main results are the following. 
Theorem 1.1. Let $a>0$. Then problem $(P)$ has a unique global solution $u(., a, \alpha, \beta)$. Moreover,

$$
\left(\left|u^{\prime}\right|^{p-2} u^{\prime}\right)^{\prime}(0)=\frac{-\alpha a^{q}}{N} .
$$

Theorem 1.2. Assume $\frac{\alpha}{\beta}<N$. Let $u$ be a solution of problem $(P)$. Then, $u$ is a decaying solution and has one of the following asymptotic behaviors.

$$
\begin{aligned}
\text { (i) If } \frac{\alpha}{q \beta}< & \frac{p}{q+1-p}, \\
& \lim _{r \rightarrow+\infty} r^{\frac{\alpha}{q \beta}} u(r)=L_{1}>0
\end{aligned}
$$

and

$$
\lim _{r \rightarrow+\infty} r^{\frac{\alpha}{q \beta}+1} u^{\prime}(r)=\frac{-\alpha}{q \beta} L_{1}<0 .
$$

(ii) If $\frac{\alpha}{q \beta}=\frac{p}{q+1-p}$,

$\lim _{r \rightarrow+\infty} r^{\frac{\alpha}{q \beta}} u(r)(\ln (r))^{\frac{-1}{q+1-p}}=$

$\left(\left(\frac{p-1}{q \beta}\right)(q+1-p)\left(\frac{N-p}{p-1}-\frac{\alpha}{q \beta}\right)\left(\frac{\alpha}{q \beta}\right)^{p-1}\right)^{\frac{1}{q+1-p}}$

(iii) If $\frac{\alpha}{q \beta}>\frac{p}{q+1-p}$,

$$
\lim _{r \rightarrow+\infty} r^{\frac{p}{q+1-p}} u(r)=
$$$$
\left(\frac{(p-1)\left(\frac{p}{q+1-p}\right)^{p-1}\left(\frac{N-p}{p-1}-\frac{p}{q+1-p}\right)}{\alpha-q \beta \frac{p}{q+1-p}}\right)^{\frac{1}{q+1-p}}
$$

and

$\lim _{r \rightarrow+\infty} r^{\frac{p}{q+1-p}+1} u^{\prime}(r)=$

$\frac{-p}{q+1-p}\left(\frac{(p-1)\left(\frac{p}{q+1-p}\right)^{p-1}\left(\frac{N-p}{p-1}-\frac{p}{q+1-p}\right)}{\alpha-q \beta \frac{p}{q+1-p}}\right)$

Now, we consider the problem

$$
\text { (Q) }\left\{\begin{array}{l}
v_{t}=\Delta_{p} v^{m} \\
v(0,1)=b
\end{array} \quad \text { in } \quad \mathbb{R}^{N} \times(0,+\infty)\right.
$$

where $p>2, N \geq 1,0<m<\frac{1}{p-1}$ and $b>0$.

Theorem 1.3. Assume $0<\frac{\alpha}{\beta}<N$ and $\frac{\alpha}{q \beta}<$ $\frac{p}{q+1-p}$. Then for every $b>0$, problem
$(Q)$ admits a radial strictly positive self-similar solution $U_{b}(x, t)=t^{-\alpha} u^{\frac{1}{m}}\left(t^{-\beta}|x|\right)$, where $\alpha=$ $\frac{\beta p-1}{1-m(p-1)}$ and $u$ is solution of problem $(P)$. Moreover, there exists $L(b)>0$ such that

$$
\lim _{t \rightarrow 0^{+}} U_{b}(x, t)=L(b)|x|^{\frac{-\alpha}{\beta}} \quad \text { for each } x \neq 0 .
$$

The paper is organized as follows. Section 2 is devoted to existence and uniqueness of global solutions of problem $(P)$, more precisely we give the proof of Theorem 1.1. In section 3, we present fundamental properties of solution $u$ of problem $(P)$ and we study also the monotonicity and behavior of $r^{c} u(r)$ where $c$ is a positive constant that we compare with the values $\frac{\alpha}{q \beta}, \frac{N-p}{p-1}$ and $\frac{p}{q+1-p}$. In section 4 we prove existence of decaying solutions of problem $(P)$ and we describe their asymptotic behavior as $r \rightarrow+\infty$ in the three cases, $\frac{\alpha}{q \beta}<\frac{p}{q+1-p}, \frac{\alpha}{q \beta}=\frac{p}{q+1-p}$ and $\frac{\alpha}{q \beta}>\frac{p}{q+1-p}$. The obtained results prove the Theorem 1.2. Finally, in section 5 we give the proof of Theorem 1.3 by applying the obtained results in the previous sections related to the parabolic equation (2).

\section{Existence of Global Solutions}

In this section, we establish the existence of global solutions of problem $(P)$.

Theorem 2.1. Let $a>0$. Then problem $(P)$ has a unique global solution $u(., a, \alpha, \beta)$. Moreover,

$$
\left(\left|u^{\prime}\right|^{p-2} u^{\prime}\right)^{\prime}(0)=\frac{-\alpha a^{q}}{N} .
$$

Proof. The proof will be done in three steps.

Step 1: Existence of a local solution.

Multiply equation (7) by $r^{N-1}$, we obtain

$\left(r^{N-1}\left|u^{\prime}\right|^{p-2} u^{\prime}+\beta r^{N}|u|^{q-1} u\right)^{\prime}=(\beta N-\alpha) r^{N-1}|u|^{q-1} u$.

Integrating (15) twice from 0 to $r$ and taking into account (8), we see that problem $(P)$ is equivalent to the equation

$$
u(r)=a-\int_{0}^{r} G(F[u](s)) d s,
$$

where

$$
G(s)=|s|^{(2-p) /(p-1)} s, \quad s \in \mathbb{R}
$$


and the nonlinear mapping $F$ is given by the formula

$$
\begin{aligned}
F[u](s)= & \beta s|u|^{q-1} u(s)+ \\
& (\alpha-\beta N) s^{1-N} \int_{0}^{s} \sigma^{N-1}|u|^{q-1} u(\sigma) d \sigma .
\end{aligned}
$$

Now we consider for $a>M>0$, the complete metric space

$$
E_{a, M, R}=\left\{\varphi \in C([0, R]):\|\varphi-a\|_{0} \leq M\right\} .
$$

Next we define the mapping $\Psi$ on $E_{a, M, R}$ by

$$
\Psi[\varphi](r)=a-\int_{0}^{r} G(F[\varphi](s)) d s .
$$

Claim 1. $\Psi$ maps $E_{a, M, R}$ into itself for some small $M$ and $R>0$.

Obviously $\Psi[\varphi] \in C([0, R])$. From the definition of the space $E_{a, M, R}, \varphi(r) \in[a-M, a+M]$, for any $r \in[0, R]$. Simple calculations show that for small $M, F[\varphi]$ has a constant sign in $[0, R]$ for every $\varphi \in$ $E_{a, M, R}$. More precisely,

$$
F[\varphi](s) \geq K s \quad \text { for all } s \in[0, R]
$$

where $K=\frac{\alpha}{2 N} a^{q}$.

Taking into account that the function $r \rightarrow \frac{G(r)}{r}$ is decreasing on $(0,+\infty)$, we have

$$
\begin{aligned}
|\Psi[\varphi](r)-a| & \leq \int_{0}^{r} \frac{G(F[\varphi](s))}{F[\varphi](s)}|F[\varphi](s)| d s \\
& \int_{0}^{r} \frac{G(K s)}{K s}|F[\varphi](s)| d s
\end{aligned}
$$

for $r \in[0, R]$. On the other hand,

$|F[\varphi](s)| \leq C s$, where $C=\left[\beta+\left|\frac{\alpha}{N}-\beta\right|\right](a+M)^{q}$.

We thus get

$$
|\Psi[\varphi](r)-a| \leq \frac{p-1}{p} C K^{\frac{2-p}{p-1}} r^{\frac{p}{p-1}}
$$

for every $r \in[0, R]$. Choose $R$ small enough such that

$$
|\Psi[\varphi](r)-a| \leq M, \quad \varphi \in E_{a, M, R} .
$$

And thereby $\Psi[\varphi] \in E_{a, M, R}$. The claim is thus proved.
Claim 2. $\Psi$ is a contraction in some interval $\left[0, r_{a}\right]$. According to Claim 1, if $r_{a}$ is a small enough, the space $E_{a, M, r_{a}}$ applies into itself. For such $r_{a}$ and any $\varphi, \psi \in E_{a, M, r_{a}}$ we have

$|\Psi[\varphi](r)-\Psi[\psi](r)| \leq \int_{0}^{r}|G(F[\varphi](s))-G(F[\psi](s))| d s$

where $F[\varphi]$ is given by (피). Next, let

$$
\Phi(s)=\min (F[\varphi](s), F[\psi](s)) .
$$

As a consequence of estimate (21), we have

$$
\Phi(s) \geq K s \quad \text { for } 0 \leq s \leq r<r_{a}
$$

and then

$$
\begin{aligned}
|G(F[\varphi](s))-G(F[\psi](s))| & \leq \frac{G(\Phi(s))}{\Phi(s)}|F[\varphi](s)-F[\psi](s)| \\
& \leq \frac{G(K s)}{K s}|F[\varphi](s)-F[\psi](s)| .
\end{aligned}
$$

Moreover,

$$
|F[\varphi](s)-F[\psi](s)| \leq C^{\prime}\|\varphi-\psi\|_{0} s,
$$

where

$$
C^{\prime}=q\left[\beta+\left|\frac{\alpha}{N}-\beta\right|\right](a+M)^{q-1} .
$$

Combining (22), (23) and (24), we have

$$
|\Psi[\varphi](s)-\Psi[\psi](s)| \leq \frac{p-1}{p} C^{\prime} K^{\frac{2-p}{p-1}} r^{\frac{p}{p-1}}\|\varphi-\psi\|_{0}
$$

for any $r \in\left[0, r_{a}\right]$. Choosing $r_{a}$ small enough, $\Psi$, is a contraction. This proves the claim.

The Banach Fixed Point Theorem then implies the existence of a unique fixed point of $\Psi$ in $E_{a, M, r_{A}}$, which is a solution of (16) and, consequently, of problem $(P)$. As usual, this solution can be extended to a maximal interval $\left[0, r_{\max }\left[, 0<r_{\max } \leq+\infty\right.\right.$.

Step 2: Existence of a global solution.

We define the following energy function

$$
E(r)=\frac{p-1}{p}\left|u^{\prime}\right|^{p}+\frac{\alpha}{q+1}|u|^{q+1}(r) .
$$

According to equation (7), we get

$$
E^{\prime}(r)=-r u^{\prime 2}\left[\frac{N-1}{r^{2}}\left|u^{\prime}\right|^{p-2}+q \beta|u|^{q-1}(r)\right] .
$$

Since $N \geq 1$ and $\beta>0$ then $E$ is decreasing, hence it is bounded. Consequently, $u$ and $u^{\prime}$ are also bounded 
and the local solution constructed above can be extended to $\mathbb{R}^{+}$.

Step 3: $\left(\left|u^{\prime}\right|^{p-2} u^{\prime}\right)^{\prime}(0)=\frac{-\alpha a^{q}}{N}$.

Integrating (15) between 0 and $r$, we get

$$
\begin{aligned}
\frac{\left|u^{\prime}\right|^{p-2} u^{\prime}}{r}= & -\beta|u|^{q-1} u(r)+ \\
& (\beta N-\alpha) r^{-N} \int_{0}^{r} s^{N-1}|u|^{q-1} u(s) d s .
\end{aligned}
$$

Hence using L'Hopital's rule and letting $r \rightarrow 0$, we obtain the desired result. The proof of Theorem is complete.

\section{Fundamental Properties}

Proposition 3.1. Assume $N>1$. Let $u$ be a solution of problem $(P)$. Then,

$$
\lim _{r \rightarrow+\infty} u(r)=\lim _{r \rightarrow+\infty} u^{\prime}(r)=0 .
$$

Proof. We show that $\lim _{r \rightarrow+\infty} E(r)=0$. Since $E^{\prime}(r) \leq 0$ and $E(r) \geq 0$ for all $r>0$, there exists a constant $l \geq 0$ such that $\lim _{r \rightarrow+\infty} E(r)=l \geq 0$.

Suppose $l>0$. Then, there exists $r_{1}>0$, such that

$$
E(r) \geq \frac{l}{2} \quad \text { for } \quad r \geq r_{1} .
$$

Now consider the function

$$
\begin{aligned}
D(r)= & E(r)+\frac{N-1}{2 r}\left|u^{\prime}\right|^{p-2} u^{\prime}(r) u(r)+ \\
& \frac{q \beta(N-1)}{2(q+1)}|u|^{q+1}(r) .
\end{aligned}
$$

Then

$$
\begin{aligned}
& D^{\prime}(r)=-q \beta r|u|^{q-1}(r) u^{\prime 2}- \\
& \quad \frac{N-1}{2 r}\left[\left|u^{\prime}\right|^{p}+\frac{N}{r}\left|u^{\prime}\right|^{p-2} u^{\prime} u+\alpha|u|^{q+1}(r)\right] .
\end{aligned}
$$

Since $\beta>0$, we have

$$
D^{\prime}(r) \leq-\frac{N-1}{2 r}\left[\left|u^{\prime}\right|^{p}+\alpha|u|^{q+1}(r)+\frac{N}{r}\left|u^{\prime}\right|^{p-2} u^{\prime} u\right] .
$$

Recalling that $u$ and $u^{\prime}$ are bounded (because $E$ is bounded), we have

$$
\lim _{r \rightarrow+\infty} \frac{\left|u^{\prime}\right|^{p-2} u^{\prime} u(r)}{r}=0 .
$$

Moreover, by (26) and (29) we have

$$
\left|u^{\prime}\right|^{p}+\alpha|u|^{q+1}(r) \geq E(r) \geq \frac{l}{2} \quad \text { for } \quad r \geq r_{1} .
$$

Consequently, there exist two constants $c>0$ and $r_{2} \geq r_{1}$ such that

$$
D^{\prime}(r) \leq-\frac{c}{r} \quad \text { for } \quad r \geq r_{2} .
$$

Integrating this last inequality between $r_{2}$ and $r$, we get

$$
D(r) \leq D\left(r_{2}\right)-c \ln \left(\frac{r}{r_{2}}\right) \quad \text { for } \quad r \geq r_{2} .
$$

In particular, we obtain $\lim _{r \rightarrow+\infty} D(r)=-\infty$. Since

$$
E(r)+\frac{N-1}{2 r}\left|u^{\prime}\right|^{p-2} u^{\prime}(r) u(r) \leq D(r),
$$

we get $\lim _{r \rightarrow+\infty} E(r)=-\infty$. This is impossible, hence the conclusion.

Proposition 3.2. Let $u$ be a solution of problem $(P)$ and let $S_{u}:=\{r>0: u(r)>0\}$. Then $u^{\prime}(r)<0$ for any $r \in S_{u}$.

Proof. We argue by contradiction. Let $r_{0}>0$ be the first zero of $u^{\prime}$. Since by (14) $u^{\prime}(r)<0$ for $r \sim 0$, we have by continuity and the definition of $r_{0}$, there exists a left neighborhood $] r_{0}-\varepsilon, r_{0}[$ (for some $\varepsilon>0$ ) where $u^{\prime}$ is strictly increasing and strictly negative, that is $\left(\left|u^{\prime}\right|^{p-2} u^{\prime}\right)^{\prime}(r)>0$ for any $\left.r \in\right] r_{0}-\varepsilon, r_{0}[$, hence by letting $r \rightarrow r_{0}$ we get $\left(\left|u^{\prime}\right|^{p-2} u^{\prime}\right)^{\prime}\left(r_{0}\right) \geq$ 0 . But by equation (7), we have $\left(\left|u^{\prime}\right|^{p-2} u^{\prime}\right)^{\prime}\left(r_{0}\right)=$ $-\alpha|u|^{q-1} u\left(r_{0}\right)<0$ since $u\left(r_{0}\right)>0, u^{\prime}\left(r_{0}\right)=0$ and $\alpha>0$. This is a contradiction. The proof is complete.

Proposition 3.3. Let $u$ be a strictly positive solution of problem $(P)$, then $u$ and $u^{\prime}$ have the same behavior (28).

Proof. If $N>1$, then by Proposition 3.1, $\lim _{r \rightarrow+\infty} u(r)=\lim _{r \rightarrow+\infty} u^{\prime}(r)=0$.

If $N=1$. Let

$$
\phi(r)=\left|u^{\prime}\right|^{p-2} u^{\prime}(r)+\beta r|u|^{q-1} u(r) .
$$

Then by equation (7),

$$
\phi^{\prime}(r)=(\beta-\alpha)|u|^{q-1} u(r) .
$$

Since $u$ is strictly positive then it is strictly decreasing. Therefore $\lim _{r \rightarrow+\infty} u(r) \in[0,+\infty[$. Suppose that, $\lim _{r \rightarrow+\infty} u(r)=L>0$. Since the energy function $E$ given by (26) converges, then necessarily, $\lim _{r \rightarrow+\infty} u^{\prime}(r)=0$. Therefore $\lim _{r \rightarrow+\infty} \phi(r)=+\infty$.

Using L'Hopital's rule, we have

$$
\lim _{r \rightarrow+\infty} \phi^{\prime}(r)=\lim _{r \rightarrow+\infty} \frac{\phi(r)}{r} .
$$


That is

$$
(\beta-\alpha) L^{q}=\beta L^{q} .
$$

Therefore, $-\alpha L^{q}=0$. But This contradicts the fact that $L>0$. Hence, $\lim _{r \rightarrow+\infty} u(r)=0$.

Now for any $c>0$, define the function

$$
E_{c}(r)=c u(r)+r u^{\prime}(r), \quad r>0 .
$$

It is clear that

$$
\left(r^{c} u(r)\right)^{\prime}=r^{c-1} E_{c}(r), \quad r>0 .
$$

Hence, using (7), we have for any $r>0$ such that $u^{\prime}(r) \neq 0$,

$$
\begin{aligned}
&(p-1)\left|u^{\prime}\right|^{p-2} E_{c}^{\prime}(r)=(p-1)\left(c-\frac{N-p}{p-1}\right)\left|u^{\prime}\right|^{p-2} u^{\prime}- \\
& \alpha r|u|^{q-1} u-q \beta r^{2}|u|^{q-1} u^{\prime}(r) \\
&=(p-1)\left(c-\frac{N-p}{p-1}\right)\left|u^{\prime}\right|^{p-2} u^{\prime}(r)- \\
& q \beta r|u|^{q-1} E_{\frac{\alpha}{q \beta}}(r) .
\end{aligned}
$$

Consequently, if $E_{c}\left(r_{0}\right)=0$ for some $r_{0}>0$, equation (7) gives

$$
\begin{aligned}
& (p-1)\left|u^{\prime}\right|^{p-2}\left(r_{0}\right) E_{c}^{\prime}\left(r_{0}\right)=r_{0}|u|^{q-1} u\left(r_{0}\right)[(q \beta c-\alpha)+ \\
& \left.(p-1) c^{p-1}\left(\frac{N-p}{p-1}-c\right) \frac{|u|^{p-q-1}\left(r_{0}\right)}{r_{0}^{p}}\right] .
\end{aligned}
$$

From which the sign of $E_{c}(r)$ for large $r$ can be obtained.

Lemma 3.4. Let $u$ be a strictly positive solution of problem $(P)$. Then $E_{c}(r) \neq 0$ for large $r$ in the following cases.

(i) $c=\frac{\alpha}{q \beta} \neq \frac{N-p}{p-1}$.

(ii) $c \neq \frac{\alpha}{q \beta}$ and $q \leq p-1$.

(iii) $c \neq \frac{\alpha}{q \beta}, q>p-1$ and $\lim _{r \rightarrow+\infty} r^{\frac{p}{q+1-p}} u(r)=$ $+\infty$.

(iv) $c \neq \frac{N-p}{p-1}, q>p-1$ and $\lim _{r \rightarrow+\infty} r^{\frac{p}{q+1-p}} u(r)=$ 0.

Proof. Assume that there exists a large $r_{0}$ such that $E_{c}\left(r_{0}\right)=0$. Using the fact that $u>0, \lim _{r \rightarrow+\infty} u(r)=$ 0 , then according to $(37)$ and our hypotheses, we get $E_{c}^{\prime}\left(r_{0}\right) \neq 0$ and thereby $E_{c}(r) \neq 0$ for large $r$.

Lemma 3.5. Assume $0<c<\frac{\alpha}{q \beta}$. Let u be a strictly positive solution of problem $(P)$. If $q \leq p-1$ or $q>$ $p-1$ and $\lim _{r \rightarrow+\infty} r^{\frac{p}{q+1-p}} u(r)=+\infty$, then $E_{c}(r)<0$ for large $r$ and $\lim _{r \rightarrow+\infty} r^{c} u(r)=0$
Proof. We know by Lemma 3.4, that $E_{c}(r) \neq 0$ for large $r$. Suppose that $E_{c}(r)>0$ for large $r$, hence

$$
r\left|u^{\prime}(r)\right|<c u(r) \quad \text { for large } r .
$$

Using this last inequality and the fact that $u>0$, we obtain according to (7)

$\left(\left|u^{\prime}\right|^{p-2} u^{\prime}\right)^{\prime}(r)<u^{q}\left[(q \beta c-\alpha)+(N-1) c^{p-1} \frac{u^{p-1-q}}{r^{p}}\right]$

If $q \leq p-1$ or $q>p-1$ and $\lim _{r \rightarrow+\infty} r^{\frac{p}{q+1-p}} u(r)=$ $+\infty$, we have $\lim _{r \rightarrow+\infty} \frac{u^{p-1-q}}{r^{p}}=0$. Then, $\left(\left|u^{\prime}\right|^{p-2} u^{\prime}\right)^{\prime}(r) \underset{+\infty}{\sim}(q \beta c-\alpha) u^{q}(r)<0$. Since $u^{\prime}(r)<0$, then $\lim _{r \rightarrow+\infty}\left|u^{\prime}\right|^{p-2} u^{\prime}(r) \in[-\infty, 0[$, but this contradicts the fact that $\lim _{r \rightarrow+\infty} u^{\prime}(r)=0$. Then, $E_{c}(r)<0$ for large $r$ and $\lim _{r \rightarrow+\infty} r^{c} u(r) \in$ $\left[0,+\infty\left[\right.\right.$. Suppose that $\lim _{r \rightarrow+\infty} r^{c} u(r)>0$, then $\lim _{r \rightarrow+\infty} r^{c+\varepsilon} u(r)=+\infty$ for $0<c+\varepsilon<\frac{\alpha}{q \beta}$. This is impossible, and therefore $\lim _{r \rightarrow+\infty} r^{c} u(r)=0$. The proof of lemma is complete.

Lemma 3.6. Assume $\frac{N-p}{p-1} \geq \frac{\alpha}{q \beta}$. Let $u$ be a strictly positive solution of problem $(P)$. Then $E_{\frac{\alpha}{q \beta}}(r)>0$ for any $r>0$.

Proof. We distinguish two cases.

Case 1. $\frac{N-p}{p-1}>\frac{\alpha}{q \beta}$.

We have $E_{\frac{\alpha}{q \beta}}(0)=\frac{\alpha}{q \beta} u(0)>0$. Let $r_{0}>0$ be the first zero of $E_{\frac{\alpha}{q \beta}}(r)$. Therefore $E_{\frac{\alpha}{q \beta}}(r)>0$ in [0, $r_{0}$ [, $E_{\frac{\alpha}{q \beta}}\left(r_{0}\right)=0$ and $E_{\frac{\alpha}{q \beta}}^{\prime}\left(r_{0}\right) \leq 0$. But using the fact that $u\left(r_{0}\right)>0$ and $\frac{\stackrel{q \beta}{N}-p}{p-1}>\frac{\alpha}{q \beta}$, we have by (37), $E_{\frac{\alpha}{q \beta}}^{\prime}\left(r_{0}\right)>0$, which is a contradiction.

Case 2. $\frac{N-p}{p-1}=\frac{\alpha}{q \beta}$.

we have by (36),

$$
(p-1)\left|u^{\prime}\right|^{p-2} E_{\frac{\alpha}{q \beta}}^{\prime}(r)=-q \beta r|u|^{q-1} E_{\frac{\alpha}{q \beta}}(r) .
$$

Let $r_{0}>0$. We introduce the following function

$$
f(r)=\frac{q \beta}{p-1} \int_{r_{0}}^{r} s\left|u^{\prime}\right|^{2-p}(s)|u|^{q-1}(s) d s .
$$

By (40), we obtain

$$
E_{\frac{\alpha}{q \beta}}^{\prime}(r)+f^{\prime}(r) E_{\frac{\alpha}{q \beta}}(r)=0 .
$$


Hence,

$$
\left(e^{f(r)} E_{\frac{\alpha}{q \beta}}(r)\right)^{\prime}=0 .
$$

Integrating this last equality from $r_{0}$ to $r$, we obtain

$$
E_{\frac{\alpha}{q \beta}}(r)=E_{\frac{\alpha}{q \beta}}\left(r_{0}\right) e^{-f(r)} \quad \forall r>r_{0} .
$$

Since $E_{\frac{\alpha}{q \beta}}\left(r_{0}\right)>0$ for any $r_{0}>0$ close to 0 , then $E_{\frac{\alpha}{q \beta}}(r) \stackrel{p \beta}{>}$ for any $r>0$.

This completes the proof of lemma.

Lemma 3.7. Assume $0<\frac{N-p}{p-1}<\frac{\alpha}{q \beta}$ and $E_{\frac{\alpha}{q \beta}}(r)>0$ for large $r$. Let $u$ be a strictly positive solution of problem $(P)$. Then $E_{\frac{N-p}{p-1}}(r)>0$ for any $r>0$.

Proof. We have $E_{\frac{N-p}{p-1}}(0)>0$. Suppose that there exists $r_{0}>0$ the first zero of $E_{\frac{N-p}{p-1}}$. Then, by 37, $E_{\frac{N-p}{p-1}}^{\prime}\left(r_{0}\right)<0$. Therefore, $E_{\frac{N-p}{p-1}}(r)<0 \forall r>$ $r_{0}$. On the other hand, since $E_{\frac{\alpha}{q \beta}}(r)>0$ for large $r$, then by (36), we have $E_{\frac{N-p}{p-1}}^{\prime}(r)<0$ for large $r$. Hence, $\lim _{r \rightarrow+\infty} E_{\frac{N-p}{p-1}}(r) \in[-\infty, 0[$, which implies that $\lim _{r \rightarrow+\infty} r u^{\prime}(r) \in[-\infty, 0[$, but this contradicts the fact that $\lim _{r \rightarrow+\infty} u(r)=0$. Consequently, $E_{\frac{N-p}{p-1}}(r)>0$ for any $r>0$.

Proposition 3.8. Assume $\frac{\alpha}{\beta}<N, \frac{\alpha}{q \beta}=\frac{p}{q+1-p}$ and $\lim _{r \rightarrow+\infty} r^{\frac{p}{q+1-p}} u(r)=+\infty$. Let $u$ be a strictly positive solution of problem $(P)$. Then

$$
\lim _{r \rightarrow+\infty} \frac{r u^{\prime}(r)}{u(r)}=\frac{-\alpha}{q \beta} .
$$

Proof. Since $\frac{\alpha}{\beta}<N$ and $\frac{\alpha}{q \beta}=\frac{p}{q+1-p}$, then $\frac{N-p}{p-1}>\frac{N}{q}>\frac{\alpha}{q \beta}$, therefore using the fact that $E_{\frac{\alpha}{q \beta}}(r)>0$ for any $r>0$ by lemma 3.6, we obtain

$$
\frac{-\alpha}{q \beta} u(r)<r u^{\prime}(r)<0 \quad \text { for any } r>0 .
$$

Let $c>0$ and

$$
g(r)=\frac{E_{c}(r)}{u(r)}=c+\frac{r u^{\prime}(r)}{u(r)}, \quad r>0 .
$$

then

$$
c-\frac{\alpha}{q \beta}<g(r)<c \quad \text { for any } r>0 .
$$

Consequently $g$ is bounded for large $r$. We prove that $g$ converges. Assume by contradiction that it oscillates, that is there exist two sequences $\left\{\eta_{i}\right\}$ and $\left\{\xi_{i}\right\}$ going to $+\infty$ as $i \rightarrow+\infty$ such that $g$ has a local minimum in $\eta_{i}$ and a local maximum in $\xi_{i}$ satisfying $\eta_{i}<\xi_{i}<\eta_{i+1}$ and

$$
\begin{aligned}
& \liminf _{r \rightarrow+\infty} g(r)=\lim _{i \rightarrow+\infty} g\left(\eta_{i}\right)=\gamma_{1}< \\
& \limsup _{r \rightarrow+\infty} g(r)=\lim _{i \rightarrow+\infty} g\left(\xi_{i}\right)=\gamma_{2} .
\end{aligned}
$$

Therefore, by (48), we have

$$
c-\frac{\alpha}{q \beta} \leq \gamma_{1}<\gamma_{2} \leq c .
$$

Since $g^{\prime}\left(\xi_{i}\right)=0$, then

$$
\frac{E_{c}^{\prime}\left(\xi_{i}\right)}{u^{\prime}\left(\xi_{i}\right)}=\frac{E_{c}\left(\xi_{i}\right)}{u\left(\xi_{i}\right)}=g\left(\xi_{i}\right) .
$$

Therefore

$$
\lim _{i \rightarrow+\infty} \frac{E_{c}^{\prime}\left(\xi_{i}\right)}{u^{\prime}\left(\xi_{i}\right)}=\gamma_{2} .
$$

On the other hand, we have by (36) and the fact that $u^{\prime}(r)<0$,

$$
\begin{aligned}
\frac{E_{c}^{\prime}(r)}{u^{\prime}(r)}= & \left(c-\frac{N-p}{p-1}\right)+ \\
& \frac{q \beta}{p-1} \frac{r u^{q}(r)}{\left|u^{\prime}\right|^{p-1}(r)}\left[\frac{\alpha}{q \beta}+\frac{r u^{\prime}(r)}{u(r)}\right] .
\end{aligned}
$$

As $E_{\frac{\alpha}{q \beta}}(r)>0 \forall r>0$, then

$$
\frac{\left|u^{\prime}(r)\right|^{p-1}}{r u^{q}(r)}<\left(\frac{\alpha}{q \beta}\right)^{p-1} r^{-p} u^{p-1-q} .
$$

Since $\lim _{r \rightarrow+\infty} r^{\frac{p}{q+1-p}} u(r)=+\infty$, then

$$
\lim _{r \rightarrow+\infty} \frac{r u^{q}(r)}{\left|u^{\prime}(r)\right|^{p-1}}=+\infty .
$$

Moreover, we have

$$
\begin{aligned}
\lim _{i \rightarrow+\infty}\left(\frac{\alpha}{q \beta}+\frac{\xi_{i} u^{\prime}\left(\xi_{i}\right)}{u\left(\xi_{i}\right)}\right) & =\frac{\alpha}{q \beta}+\lim _{i \rightarrow+\infty} g\left(\xi_{i}\right)-c \\
& =\frac{\alpha}{q \beta}+\gamma_{2}-c>0 .
\end{aligned}
$$

Then, by (53)

$$
\lim _{i \rightarrow+\infty} \frac{E_{c}^{\prime}\left(\xi_{i}\right)}{u^{\prime}\left(\xi_{i}\right)}=+\infty .
$$


But this contradicts (52). Then $g(r)$ converges as $r \rightarrow$ $+\infty$, and consequently $\frac{r u^{\prime}(r)}{u(r)}$ converges also. Let $\lim _{r \rightarrow+\infty} \frac{r u^{\prime}(r)}{u(r)}=-d \leq 0$, then by (46), $0 \leq d \leq \frac{\alpha}{q \beta}$. Suppose that $d<\frac{\alpha}{q \beta}$, then

$$
\lim _{r \rightarrow+\infty}\left(\frac{\alpha}{q \beta}+\frac{r u^{\prime}(r)}{u(r)}\right)=\frac{\alpha}{q \beta}-d>0 .
$$

Therefore, by (53) and (55),

$$
\lim _{r \rightarrow+\infty} \frac{E_{c}^{\prime}(r)}{u^{\prime}(r)}=+\infty
$$

Using Hospital's rule, we get

$$
\begin{aligned}
\lim _{r \rightarrow+\infty} \frac{E_{c}^{\prime}(r)}{u^{\prime}(r)}=\lim _{r \rightarrow+\infty} \frac{E_{c}(r)}{u(r)} & =\lim _{r \rightarrow+\infty}\left(c+\frac{r u^{\prime}(r)}{u(r)}\right) \\
& =c-d .
\end{aligned}
$$

This contradicts (59). Consequently $\lim _{r \rightarrow+\infty} \frac{r u^{\prime}(r)}{u(r)}=$ $\frac{-\alpha}{q \beta}$. The proof is complete.

Proposition 3.9. Assume $\frac{\alpha}{\beta}<N, \frac{\alpha}{q \beta}=\frac{p}{q+1-p}$ and $\lim _{r \rightarrow+\infty} r^{\frac{p}{q+1-p}} u(r)=+\infty$. Let $u$ be a strictly positive solution of problem $(P)$. Then,

- if $0<c<\frac{\alpha}{q \beta}, \lim _{r \rightarrow+\infty} r^{c} u(r)=$ $\lim _{r \rightarrow+\infty} r^{c+1} u^{\prime}(r)=0$.

- if $c>\frac{\alpha}{q \beta}, \lim _{r \rightarrow+\infty} r^{c} u(r)=+\infty$ and $\lim _{r \rightarrow+\infty} r^{c+1} u^{\prime}(r)=-\infty$.

Proof. First, we show that $E_{c}^{\prime}(r) \neq 0$ for large $r$. If $E_{c}^{\prime}(r)=0$ for some large $r$, then

$$
\begin{aligned}
& (p-1)\left|u^{\prime}\right|^{p-2} E_{c}^{\prime \prime}(r)=r u^{q-1}\left|u^{\prime}\right|\left[q \beta\left(\frac{\alpha}{q \beta}-c\right)-\right. \\
& q \beta(q-1) \frac{E_{\frac{\alpha}{q \beta}} E_{\frac{1}{q-1}}}{r\left|u^{\prime}\right| u}-q \beta(p-1)\left(c-\frac{N-p}{p-1}\right) \frac{E_{\frac{\alpha}{q \beta}}}{r\left|u^{\prime}\right|}+ \\
& \left.(p-1)(N-1)\left(c-\frac{N-p}{p-1}\right) \frac{\left|u^{\prime}\right|{ }^{p-1}}{r u^{q}} \frac{u}{r\left|u^{\prime}\right|}\right]
\end{aligned}
$$

We know by Proposition 3.8 that $\lim _{r \rightarrow+\infty} \frac{r u^{\prime}(r)}{u(r)}=$ $\frac{-\alpha}{q \beta}$, then

$$
\lim _{r \rightarrow+\infty} \frac{E_{\frac{1}{q-1}}(r)}{u(r)}=\frac{1}{q-1}-\frac{\alpha}{q \beta}
$$

and

$$
\lim _{r \rightarrow+\infty} \frac{E_{\frac{\alpha}{q \beta}}(r)}{r\left|u^{\prime}\right|}=0 .
$$

On the other hand, since $E_{\frac{\alpha}{\alpha \beta}}(r)>0, \forall r>0$ (by Lemma 3.6 ) and $\lim _{r \rightarrow+\infty} r^{\frac{p^{q \beta}}{q+1-p}}=0$, we obtain

$$
\lim _{r \rightarrow+\infty} \frac{\left|u^{\prime}(r)\right|^{p-1}}{r u^{q}(r)}=0 .
$$

Therefore, using the fact that $\lim _{r \rightarrow+\infty} \frac{u(r)}{r\left|u^{\prime}\right|}=\frac{q \beta}{\alpha}$, we get

$$
\lim _{r \rightarrow+\infty} \frac{\left|u^{\prime}\right|^{p-1}(r)}{r u^{q}(r)} \frac{u(r)}{r\left|u^{\prime}(r)\right|}=0 .
$$

Using (62), (63) and (65), we get from (61),$E_{c}^{\prime \prime}(r) \neq$ 0 if $c \neq \frac{\alpha}{q \beta}$. Consequently, if $c \neq \frac{\alpha}{q \beta}$, we have $E_{c}^{\prime}(r) \neq 0$ for large $r$. We distinguish two cases.

Case 1. $0<c<\frac{\alpha}{q \beta}$.

We have by Lemma 3.5, $E_{c}(r)<0$ for large $r$ and $\lim _{r \rightarrow+\infty} r^{c} u(r)=0$. If $E_{c}^{\prime}(r)<0$ for large $r$, then $\lim _{r \rightarrow+\infty} E_{c}(r) \in[-\infty ; 0[$, this is impossible since $\lim _{r \rightarrow+\infty} u(r)=0$ and $\lim _{r \rightarrow+\infty} r u^{\prime}(r)=0$. Therefore, $E_{c}^{\prime}(r)>0$ for large $r$. On the other hand, we have

$$
\left(r^{c+1} u^{\prime}\right)^{\prime}=r^{c} E_{c}^{\prime}(r),
$$

Then the function $r^{c+1} u^{\prime}$ is negative and increasing for large $r$ and therefore, using L'Hopital's rule, we obtain $\lim _{r \rightarrow+\infty} r^{c+1} u^{\prime}(r)=\lim _{r \rightarrow+\infty} r^{c} u(r)=0$.

Case 2. $c>\frac{\alpha}{q \beta}$.

We have $E_{c}(r)>0, \forall r>0$ (by Lemma 3.6). If $E_{c}^{\prime}(r)>0$ for large $\left.\left.r, \lim _{r \rightarrow+\infty} E_{c}(r) \in\right] 0 ;+\infty\right]$, this is also impossible. Therefore, $E_{c}^{\prime}(r)<0$ for large $r$. Hence, $\left.\left.\lim _{r \rightarrow+\infty} r^{c} u(r) \in\right] 0,+\infty\right]$ and $\lim _{r \rightarrow+\infty} r^{c+1} u^{\prime}(r) \in[-\infty, 0[$. Suppose that $-\infty<$ $\lim _{r \rightarrow+\infty} r^{c+1} u^{\prime}(r)<0$, then by L'Hopital's rule, $0<$ $\lim _{r \rightarrow+\infty} r^{c} u(r)<+\infty$.

Using logarithmic change (9), we have $v_{c}$ and $h_{c}$ converge, $A_{c}>0$ and $K_{c}<0$ and by letting $t \rightarrow+\infty$ in equation (10), we obtain $\lim _{t \rightarrow+\infty} w_{c}^{\prime}(t)>0$. But this contradicts the fact that $w$ converges. Therefore $\lim _{r \rightarrow+\infty} r^{c+1} u^{\prime}(r)=-\infty$ and $\lim _{r \rightarrow+\infty} r^{c} u(r)=+\infty$. The proof is complete.

Proposition 3.10. Let $u$ be a solution of problem $(P)$. If there exists $c>0$ such that $r^{c} u(r)$ is 
monotone for large $r$ and $\lim _{r \rightarrow+\infty} r^{c} u(r)=d$. Then $\lim _{r \rightarrow+\infty} r^{c+1} u^{\prime}(r)=-c d$.

Proof. According to logarithmic change (9) and 34), we have

$$
v_{c}^{\prime}(t)=r^{c} E_{c}(r) .
$$

Then, the function $v_{c}(t)$ is monotone for large $t$ and $\lim _{t \rightarrow+\infty} v_{c}(t)=d$. Therefore for large $t_{0}$, the integral $\int_{t_{0}}^{t}\left|v_{c}^{\prime}(s)\right| d s$ converges as $t \rightarrow+\infty$. Therefore, $\lim _{t \rightarrow+\infty} v_{c}^{\prime}(t)=0$. Hence, by (12) $\lim _{t \rightarrow+\infty} h_{c}(t)=$ $-c d$, that is $\lim _{r \rightarrow+\infty} r^{c+1} u^{\prime}(r)=-c d$.

\section{Asymptotic Behavior at infinity}

In this section we study the asymptotic behavior near infinity of positive solutions of problem $(P)$.

Theorem 4.1. Assume $\frac{\alpha}{\beta}<N$. Then any solution of problem $(P)$ is a decaying solution.

Proof. We have $u(0)>0$. Assume by contradiction that there exists $r_{0}>0$ such that $u\left(r_{0}\right)=0$ (where $r_{0}$ is the first zero of $u$ ). Then, $u^{\prime}\left(r_{0}\right) \leq 0$. On the other hand, integrating (15) between 0 and $r_{0}$, we obtain

$$
r_{0}^{N-1}\left|u^{\prime}\right|^{p-2} u^{\prime}\left(r_{0}\right)=(\beta N-\alpha) \int_{0}^{r_{0}} s^{N-1} u^{q}(s) d s .
$$

The right-hand side of the previous equality is strictly positive, but this contradicts the fact that $u^{\prime}\left(r_{0}\right) \leq$ 0 . Therefore $u$ is strictly positive and therefore it is strictly decreasing by Proposition 3.2. Hence $u$ is a decaying solution. The theorem is proved.

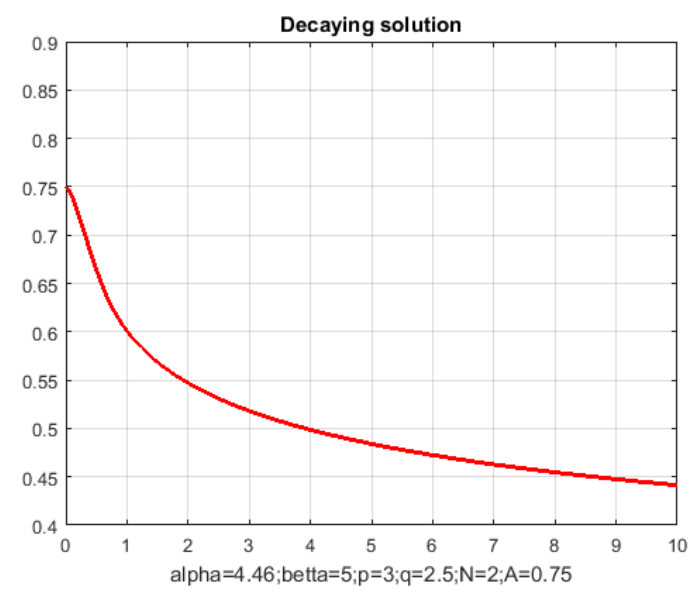

A simple calculation gives

$$
I^{\prime}(r)=\frac{-1}{\alpha}\left(N-\frac{\alpha}{\beta}\right) r^{\frac{\alpha}{\beta}-2}\left|u^{\prime}\right|^{p-2} u^{\prime}(r) .
$$

Since $N>\frac{\alpha}{\beta}$ and $u^{\prime}(r)<0$, then $I^{\prime}(r)>0 \forall r>0$. Moreover, using (14), the fact that $u(0)=a>0$, we get $\lim _{r \rightarrow 0} I(r)=0$. Therefore, $I(r)>0 \forall r>$ 0 , hence $\left.\left.\lim _{r \rightarrow+\infty} I(r) \in\right] 0,+\infty\right]$ and then there exists $c>0$ such that $I(r) \geq c$ for large $r$. As $u^{\prime}(r)<0$, then

$$
r^{\frac{\alpha}{\beta}} u^{q}(r) \geq \frac{\alpha}{\beta} c \text { for large } r .
$$

On the other hand, we know that by Lemma 3.4 and Lemma 3.6 that $E_{\frac{\alpha}{q \beta}}(r) \neq 0$ for large $r$. Then, from (73), necessarily $\left.\left.\lim _{r \rightarrow+\infty} r^{\frac{\alpha}{\alpha \beta}} u(r) \in\right] 0 ;+\infty\right]$. Suppose that $\lim _{r \rightarrow+\infty} r^{\frac{\alpha}{q \beta}} u(r)=+\infty$, then necessarily $E_{\frac{\alpha}{q \beta}}(r)>0$ for large $r$ and therefore

$$
0<\frac{\left|u^{\prime}\right|^{p-1}(r)}{r u^{q}(r)}<\left(\frac{\alpha}{q \beta}\right)^{p-1} \frac{1}{r^{p} u^{q+1-p}(r)} .
$$

As, $\lim _{r \rightarrow+\infty} r^{\frac{\alpha}{\alpha \beta}} u(r) \quad=\quad+\infty$, then $\lim _{r \rightarrow+\infty} r^{p} u^{q+1-p}(r)=+\infty$, which implies according to (74) that $\lim _{r \rightarrow+\infty} \frac{\left|u^{\prime}\right|^{p-1}(r)}{r u^{q}(r)}=0$. This leads from (71) that $I(r) \underset{+\infty}{\sim} \frac{\beta}{\alpha} r^{\frac{\alpha}{\beta}} u^{q}(r)$ and therefore $\lim _{r \rightarrow+\infty} I(r)=+\infty$. Let

$0<\sigma<\min \left(\frac{\alpha}{q \beta} ; \frac{1}{p-1}\left[\frac{\alpha}{q \beta}(p-1-q)+p\right]\right)$. 
By Lemma 3.5, we obtain, $\lim _{r \rightarrow+\infty} r^{\frac{\alpha}{q \beta}-\sigma} u(r)=0$. Then

$$
u(r) \leq r^{\sigma-\frac{\alpha}{q \beta}} \quad \text { for large } r .
$$

Using this last inequality and the fact that $E_{\frac{\alpha}{q \beta}}(r)>0$ for large $r$, we get

$$
\begin{aligned}
& 0<I^{\prime}(r)< \\
& \frac{1}{\alpha}\left(N-\frac{\alpha}{\beta}\right)\left(\frac{\alpha}{q \beta}\right)^{p-1} r^{\sigma(p-1)+\frac{\alpha}{q \beta}(q+1-p)-p-1} .
\end{aligned}
$$

Since $\sigma(p-1)+\frac{\alpha}{q \beta}(q+1-p)-p<0$, then $\lim _{r \rightarrow+\infty} I(r)$ is finite, which contradicts the fact that $\lim _{r \rightarrow+\infty} I(r)=+\infty$. Consequently, $\lim _{r \rightarrow+\infty} r^{\frac{\alpha}{q \beta}} u(r)=$ $L_{1}>0$. Moreover, since $E_{\frac{\alpha}{q \beta}}(r) \neq 0$ for large $r$, then $r^{\frac{\alpha}{q \beta}} u(r)$ is monotone for large $r$. Therefore, by Proposition 3.10, $\lim _{r \rightarrow+\infty} r^{\frac{\alpha}{q \beta}+1} u^{\prime}(r)=\frac{-\alpha}{q \beta} L_{1}<0$. This completes the proof.

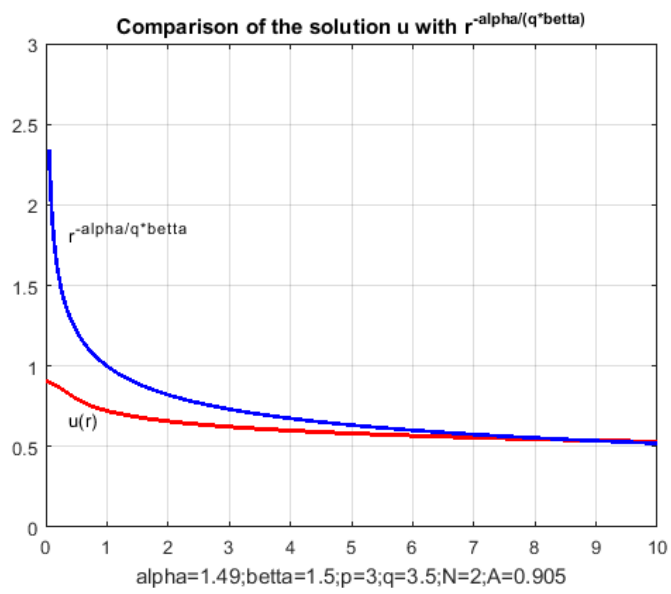

Theorem 4.3. Assume $\frac{\alpha}{\beta}<N$ and $\frac{\alpha}{q \beta}=$ $\frac{p}{q+1-p}$. Let $u$ be a solution of problem $(P)$. Then $\lim _{r \rightarrow+\infty} r^{\frac{\alpha}{q \beta}} u(r)(\ln (r))^{\frac{-1}{q+1-p}}=$

$\left(\left(\frac{p-1}{q \beta}\right)(q+1-p)\left(\frac{N-p}{p-1}-\frac{\alpha}{q \beta}\right)\left(\frac{\alpha}{q \beta}\right)^{p-1}\right)^{\frac{1}{q+1-p}}$

Proof. First we show that $\lim _{r \rightarrow+\infty} r^{\frac{\alpha}{q \beta}} u(r)=+\infty$.

Since $u$ is strictly positive, we introduce this following function

$$
\varphi(r)=r^{N-1}\left|u^{\prime}\right|^{p-2} u^{\prime}(r)+\beta r^{N} u^{q}(r) .
$$

then by (15), we get

$$
\varphi^{\prime}(r)=(\beta N-\alpha) r^{N-1} u^{q}(r) .
$$

Since $N \beta>\alpha$ and $u(r)>0$, then $\varphi^{\prime}(r)>0$ and as $\varphi(0)=0$, we have $\varphi(r)>0 \forall r>0$. That is, for any $r>0$,

$$
\left|u^{\prime}\right|^{p-2} u^{\prime}(r)>-\beta r u^{q}(r)
$$

As $u^{\prime}(r)<0$, then for any $r>0$

$$
u^{\prime}(r) u^{\frac{-q}{p-1}}>-\beta^{\frac{1}{p-1}} r^{\frac{1}{p-1}} \text {. }
$$

Integrating (80) twice from $r_{0}$ to $r$ and taking into account $q>p-1$, we obtain

$$
\begin{aligned}
& u^{\frac{p-1-q}{p-1}}(r)-u^{\frac{p-1-q}{p-1}}\left(r_{0}\right)< \\
& \frac{q+1-p}{p} \beta^{\frac{1}{p-1}}\left(r^{\frac{p}{p-1}}-r_{0}^{\frac{p}{p-1}}\right) .
\end{aligned}
$$

Then there exists $C>0$ such that

$$
r^{\frac{p}{q+1-p}} u(r)>C \text { for large } r \text {. }
$$

As $\frac{\alpha}{\beta}<N$ and $\frac{\alpha}{q \beta}=\frac{p}{q+1-p}$, then $\frac{N-p}{p-1}>$ $\frac{\alpha}{q \beta}$. Hence, by Lemma 3.6, $E_{\frac{\alpha}{q \beta}}(r)>0$ for any $r>$ 0 . Consequently $\left.\left.\lim _{r \rightarrow+\infty} r^{\frac{\alpha}{q \beta}} u(r) \in\right] 0,+\infty\right]$. Suppose that $\lim _{r \rightarrow+\infty} r^{\frac{\alpha}{q \beta}} u(r)=l>0$. Using equation (7), we get

$$
\begin{aligned}
& r^{N-1}\left|u^{\prime}\right|^{p-2} u^{\prime}(r)+\beta r^{N} u^{q}(r)= \\
& (\beta N-\alpha) \int_{0}^{r} s^{N-1} u^{q}(s) d s .
\end{aligned}
$$

Then

$$
\begin{gathered}
r^{\frac{\alpha}{\beta}-1}\left|u^{\prime}\right|^{p-2} u^{\prime}(r)+\beta r^{\frac{\alpha}{\beta}} u^{q}(r)= \\
(\beta N-\alpha) r^{\frac{\alpha}{\beta}-N} \int_{0}^{r} s^{N-1} u^{q}(s) d s .
\end{gathered}
$$

Since

$\int_{0}^{r} s^{N-1} u^{q}(s) d s>\frac{1}{N} r^{N} u^{q}=\frac{1}{N} r^{\frac{\alpha}{\beta}} u^{q} r^{N-\frac{\alpha}{\beta}} \underset{r \rightarrow+\infty}{\longrightarrow}+\infty$ Then, using L'Hopital's rule, we obtain

$$
\begin{aligned}
\int_{r \rightarrow+\infty} \frac{\int_{0}^{r} s^{N-1} u^{q}(s) d s}{r^{N-\frac{\alpha}{\beta}}} & =\lim _{r \rightarrow+\infty} \frac{r^{N-1} u^{q}(r)}{\left(N-\frac{\alpha}{\beta}\right) r^{N-\frac{\alpha}{\beta}-1}} \\
& =\frac{\beta l^{q}}{\beta N-\alpha}
\end{aligned}
$$


Therefore, from (84) we have

$$
\lim _{r \rightarrow+\infty} r^{\frac{\alpha}{\beta}-1}\left|u^{\prime}\right|^{p-2} u^{\prime}(r)=0 .
$$

that is,

$$
\lim _{r \rightarrow+\infty} r^{\frac{\alpha}{\beta}-p}+1 u^{\prime}(r)=0
$$

Using L'Hopital's rule and the fact that $\frac{\frac{\alpha}{\beta}-p}{p-1}=$ $\frac{p}{q+1-p}$, we obtain $\lim _{r \rightarrow+\infty} r^{\frac{p}{q+1-p}} u(r)=0$. But this contradicts the fact that $\lim _{r \rightarrow+\infty} r^{\frac{p}{q+1-p}} u(r)=l>$ 0. Consequently, $\lim _{r \rightarrow+\infty} r^{\frac{p}{q+1-p}} u(r)=+\infty$.

Using the fact that $u$ is strictly positive and decreasing, we obtain by (36),

$$
\begin{aligned}
& \frac{E_{\frac{\alpha}{q \beta}}^{\prime}(r)}{u^{\prime}}=\left(\frac{\alpha}{q \beta}-\frac{N-p}{p-1}\right)+\frac{q \beta}{p-1} \frac{r u^{q}}{\left|u^{\prime}\right| p-1} \frac{E_{\frac{\alpha}{q \beta}}(r)}{u}= \\
& \left(\frac{\alpha}{q \beta}-\frac{N-p}{p-1}\right)+\frac{q \beta}{p-1}\left(\frac{u}{r\left|u^{\prime}\right|}\right)^{p-1} r^{p} u^{q+1-p} \frac{E_{\frac{\alpha}{q \beta}}(r)}{u} .
\end{aligned}
$$

We introduce the following variable change

$$
V(r)=r^{\frac{\alpha}{q \beta}} u(r), \quad r>0 .
$$

It's easy to see by (35)that

$$
r V^{q-p} V^{\prime}(r)=r^{p} u^{q+1-p} \frac{E_{\frac{\alpha}{q \beta}}(r)}{u} .
$$

Then, by (88)

$$
\begin{aligned}
\frac{E_{\frac{\alpha}{q \beta}}^{\prime}(r)}{u^{\prime}} & =\left(\frac{\alpha}{q \beta}-\frac{N-p}{p-1}\right)+ \\
& \frac{q \beta}{p-1}\left(\frac{u}{r\left|u^{\prime}\right|}\right)^{p-1} r V^{q-p} V^{\prime}(r) .
\end{aligned}
$$

Using L'Hopital's rule and proposition 3.8, we get

$$
\lim _{r \rightarrow+\infty} \frac{E_{\frac{\alpha}{q \beta}}^{\prime}(r)}{u^{\prime}}=\lim _{r \rightarrow+\infty} \frac{E_{\frac{\alpha}{q \beta}}(r)}{u}=\frac{\alpha}{q \beta}+\frac{r u^{\prime}}{u}=0 .
$$

Therefore by (91)

$$
\begin{aligned}
& \lim _{r \rightarrow+\infty} r V^{q-p} V^{\prime}(r)= \\
& \frac{p-1}{q \beta}\left(\frac{N-p}{p-1}-\frac{\alpha}{q \beta}\right)\left(\frac{\alpha}{q \beta}\right)^{p-1} .
\end{aligned}
$$

That is to say

$$
\begin{gathered}
\lim _{r \rightarrow+\infty} \frac{\left(\frac{V^{q+1-p}(r)}{q+1-p}\right)^{\prime}}{(\ln (r))^{\prime}}= \\
\frac{p-1}{q \beta}\left(\frac{N-p}{p-1}-\frac{\alpha}{q \beta}\right)\left(\frac{\alpha}{q \beta}\right)^{p-1} .
\end{gathered}
$$

Using L'Hopital's rule (because $\lim _{r \rightarrow+\infty} V(r)=+\infty$ ), we get

$$
\begin{aligned}
& \lim _{r \rightarrow+\infty} \frac{V^{q+1-p}(r)}{\ln (r)}= \\
& \frac{p-1}{q \beta}(q+1-p)\left(\frac{N-p}{p-1}-\frac{\alpha}{q \beta}\right)\left(\frac{\alpha}{q \beta}\right)^{p-1} .
\end{aligned}
$$

The result follows and the proof is complete.

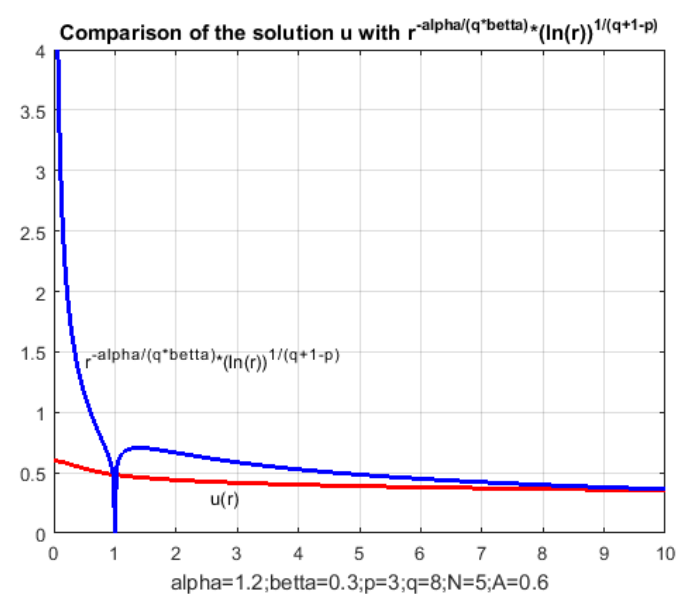

Theorem 4.4. Assume $\frac{\alpha}{\beta}<N$ and $\frac{\alpha}{q \beta}>$ $\frac{p}{q+1-p}$. Let $u$ be a solution of problem $(P)$. Then

$$
\lim _{r \rightarrow+\infty} r^{\frac{p}{q+1-p}} u(r)=L_{2}
$$

and

$$
\lim _{r \rightarrow+\infty} r^{\frac{p}{q+1-p}+1} u^{\prime}(r)=\frac{-p}{q+1-p} L_{2},
$$

where

$$
L_{2}=\left(\frac{(p-1)\left(\frac{p}{q+1-p}\right)^{p-1}\left(\frac{N-p}{p-1}-\frac{p}{q+1-p}\right)}{\alpha-q \beta \frac{p}{q+1-p}}\right)^{\frac{1}{q+1-p}} .
$$


Proof. As $\frac{\alpha}{\beta}<N$ and $\frac{\alpha}{q \beta}>\frac{p}{q+1-p}$, then $\frac{N-p}{p-1}>\frac{p}{q+1-p}$. First, we show that $E_{\frac{p}{q+1-p}}(r)>0 \forall r>0$. Let $r_{0}>0$ the first zero of $E_{\frac{p}{q+1-p}}(r)$. Then we have $E_{\frac{p}{q+1-p}}(r)>0$ $\forall r \in\left[0, r_{0}\right), E_{\frac{p}{q+1-p}}\left(r_{0}\right)=0$ and $E_{\frac{p}{q+1-p}}^{\prime q+1-p}\left(r_{0}\right) \leq 0$. Therefore using (37)

$$
\begin{aligned}
& \left(q \beta \frac{p}{q+1-p}-\alpha\right)+(p-1)\left(\frac{p}{q+1-p}\right)^{p-1} \\
& \left(\frac{N-p}{p-1}-\frac{p}{q+1-p}\right) \frac{u^{p-q-1}\left(r_{0}\right)}{r_{0}^{p}} \leq 0
\end{aligned}
$$

Hence

$$
r_{0}^{\frac{p}{q+1-p}} u\left(r_{0}\right) \geq L_{2}
$$

Where $L_{2}$ is given by (98). On the other hand, since $u(r)>0$, then integrating (15) on $\left(0, r_{0}\right)$, we obtain

$$
\begin{aligned}
& r_{0}^{N-1}\left|u^{\prime}\right|^{p-2} u^{\prime}\left(r_{0}\right)+\beta r_{0}^{N} u^{q}\left(r_{0}\right)= \\
& (\beta N-\alpha) \int_{0}^{r_{0}} s^{N-1} u^{q}(s) d s .
\end{aligned}
$$

Therefore,

$$
\begin{aligned}
\beta\left(r_{0}^{\frac{p}{q+1-p}} u\left(r_{0}\right)\right)^{q} & =r_{0}^{\frac{p q}{q+1-p}-1}\left|u^{\prime}\right|^{p-1}\left(r_{0}\right)+ \\
& (\beta N-\alpha) r_{0}^{\frac{p q}{q+1-p}-N} \int_{0}^{r_{0}} s^{N-1} u^{q}(s) d s .
\end{aligned}
$$

As $E_{\frac{p}{q+1-p}}(r)>0 \forall r \in\left[0, r_{0}\right)$ and $E_{\frac{p}{q+1-p}}\left(r_{0}\right)=$ 0 , then $\left(r^{\frac{p}{q+1-p}} u(r)\right)^{\prime}>0 \forall r \in\left(0, r_{0}\right)$ and $\left|u^{\prime}\left(r_{0}\right)\right|=\frac{p}{q+1-p} \frac{u\left(r_{0}\right)}{r_{0}}$. Then,

$$
\begin{aligned}
& \beta\left(r_{0}^{\frac{p}{q+1-p}} u\left(r_{0}\right)\right)^{q} \leq \\
& \left(\frac{p}{q+1-p}\right)^{p-1} r^{\frac{p q}{q+1-p}-p} u^{p-1}\left(r_{0}\right)+ \\
& (\beta N-\alpha) r_{0}^{\frac{p q}{q+1-p}-N} r^{\frac{p q}{q+1-p}} u^{q}\left(r_{0}\right) \int_{0}^{r_{0}} s^{N-1-\frac{p q}{q+1-p}} d s .
\end{aligned}
$$

Taking into account $N>\frac{p q}{q+1-p}$, we obtain

$$
\begin{aligned}
\beta\left(r_{0}^{\frac{p}{q+1-p}} u\left(r_{0}\right)\right)^{q} & \leq\left(\frac{p}{q+1-p}\right)^{p-1} r^{\frac{p(p-1)}{q+1-p}} u^{p-1}\left(r_{0}\right)+ \\
& \frac{\beta N-\alpha}{N-\frac{p q}{q+1-p}}\left(r_{0}^{\frac{p}{q+1-p}} u\left(r_{0}\right)\right)^{q} .
\end{aligned}
$$

Therefore

$$
\begin{aligned}
& r_{0}^{\frac{p}{q+1-p}} u\left(r_{0}\right) \leq \\
& {\left[\left(\frac{p}{q+1-p}\right)^{p-1}\left(\frac{1}{\beta-\frac{\beta N-\alpha}{N-\frac{p q}{q+1-p}}}\right)\right]^{\frac{1}{q+1-p}}=L_{2} .}
\end{aligned}
$$

Hence by (100) and (105),

$$
r_{0}^{\frac{p}{q+1-p}} u\left(r_{0}\right)=L_{2}
$$

As $E_{\frac{p}{q+1-p}}\left(r_{0}\right)=0$, then

$$
r_{0}^{\frac{p}{q+1-p}+1} u^{\prime}\left(r_{0}\right)=\frac{-p}{q+1-p} L_{2} .
$$

Recalling the logarithmic change (9), $v(t)=$ $r^{\frac{p}{q+1-p}} u(r)$, we obtain by (10), (11) and (12) and (13), the system

$$
\left\{\begin{aligned}
v^{\prime}(t)= & \frac{p}{q+1-p} v(t)+|w(t)|^{\frac{2-p}{p-1}} w(t) \\
w^{\prime}(t)= & -\left(N-p-\frac{p}{q+1-p}(p-1)\right) w(t)- \\
& \alpha v^{q}(t)-q \beta v^{q-1}(t)|w(t)|^{\frac{2-p}{p-1}} w(t) .
\end{aligned}\right.
$$

This system has a non trivial equilibrium point $\left(L_{2},-\left(\frac{p}{q+1-p} L_{2}\right)^{p-1}\right)$ and admits a unique solution, but $v\left(t_{0}\right)=L_{2}$ and $w\left(t_{0}\right) \quad=\quad-\left(\frac{p}{q+1-p} L_{2}\right)^{p-1} \quad$ (because $\left.h\left(t_{0}\right)=\frac{-p}{q+1-p} L_{2}\right)$, where $t_{0}=\ln \left(r_{0}\right)$, then necessarily $v(t)=L_{2}$ and $w(t)=$ $-\left(\frac{p}{q+1-p} L_{2}\right)^{p-1}$, therefore $v^{\prime}(t)=0$ and by (67), $E_{\frac{p}{q+1-p}}(r)=0, \forall r>0$. This is a contradiction. We deduce that $E_{\frac{p}{q+1-p}}(r)>0 \forall r>0$ and $\left.\left.\lim _{r \rightarrow+\infty} r^{\frac{p}{q+1-p}} u(r) \in\right] 0,+\infty\right]$. That is,

$$
\left|u^{\prime}(r)\right|<\frac{p}{q+1-p} \frac{u(r)}{r}, \quad \forall r>0 .
$$


In the same way, by integrating (15) on $(0, r)$ we obtain $r^{\frac{p}{q+1-p}} u(r) \leq L_{2} \forall r>0$. Then, $\left.\left.\lim _{r \rightarrow+\infty} r^{\frac{p}{q+1-p}} u(r)=d \in\right] 0, L_{2}\right]$. We show that $d=L_{2}$. According to Proposition 3.10, since $v^{\prime}(t)>0$ and $\lim _{t \rightarrow+\infty} v(t)=d>0$, then $\lim _{t \rightarrow+\infty} h(t)=\frac{-p}{q+1-p} d<0 . \quad$ Therefore, $\lim _{t \rightarrow+\infty} w(t)=-\left(\frac{p}{q+1-p} L_{2}\right)^{p-1}$ and necessarily $\lim _{t \rightarrow+\infty} w^{\prime}(t)=0$. Hence, letting $t \rightarrow+\infty$ in the second equation of system (108), we get $d=L_{2}$ and the proof is complete.

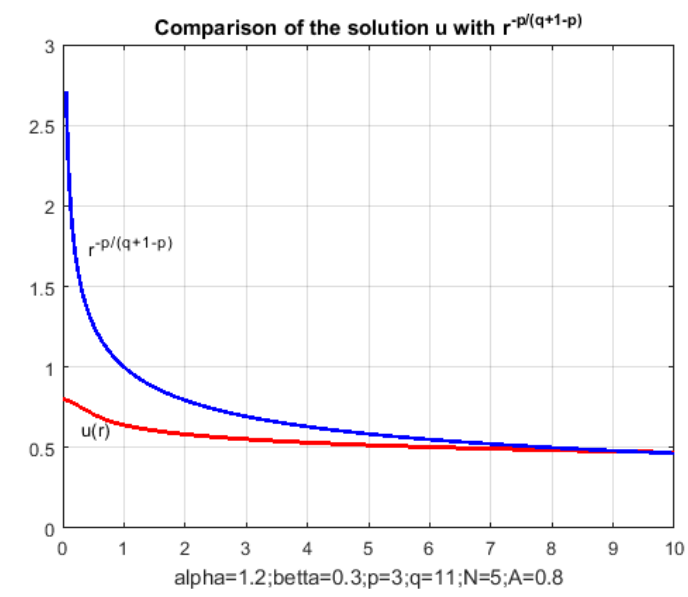

\section{Application to the parabolic problem}

In this section, we prove the existence of radial strictly positive self-similar solution of the following parabolic problem

$$
\text { (Q) }\left\{\begin{array}{l}
v_{t}=\Delta_{p} v^{m} \\
v(0,1)=b
\end{array} \quad \text { in } \quad \mathbb{R}^{N} \times(0,+\infty)\right.
$$

where $p>2, N \geq 1,0<m<\frac{1}{p-1}$ and $b>0$.

Theorem 5.1. Assume $0<\frac{\alpha}{\beta}<N$ and $\frac{\alpha}{q \beta}<$ $\frac{p}{q+1-p}$. Then, for every $b>0$, problem $(Q)$ admits a radial strictly positive self-similar solution $U_{b}(x, t)=t^{-\alpha} u^{\frac{1}{m}}\left(t^{-\beta}|x|\right)$, where $\alpha=$ $\frac{\beta p-1}{1-m(p-1)}$ and $u$ is solution of problem $(P)$. Moreover, there exists $L(b)>0$ such that

$$
\lim _{t \rightarrow 0^{+}} U_{b}(x, t)=L(b)|x|^{\frac{-\alpha}{\beta}} \quad \text { for each } x \neq 0 .
$$

Proof. The Existence and uniqueness of $U_{b}$ follow from Theorem 2.1 with $b=a^{q}$ and $m=\frac{1}{q}$. The positivity follows easily from Theorem 4.1. Put $y=t^{-\beta}|x|$, then

$$
|x|^{\frac{\alpha}{\beta}} U_{b}(x, t)=y^{\frac{\alpha}{\beta}} u^{q}(y) .
$$

According to Theorem 4.2, we have $\lim _{y \rightarrow+\infty} y^{\frac{\alpha}{\beta}} u^{q}(y)=L_{1}^{q}>0$. Therefore, there exists $L(b)=L_{1}^{q}>0$, such that

$$
\lim _{t \rightarrow 0^{+}}|x|^{\frac{\alpha}{\beta}} U_{b}(x, t)=\lim _{y \rightarrow+\infty} y^{\frac{\alpha}{\beta}} u^{q}(y)=L(b) .
$$

The proof is complete.

\section{References:}

[1] P. Aviles. Local behavior of positive solutions of some elliptic equation. Comm.Math.Phys 108 (1987) 177-192.

[2] D.G. Aronson and L.A. Peletier. Large time behaviour of solutions of the porous media equation in bounded domains. J. Diff. Eqs. 39 (1981), 378412.

[3] P. Bénilan, M.G. Crandall and M. Pierre. Solutions of the porous media equation in $\mathbb{R}^{N}$ under optimal conditions on initial values. Indiana Univ. Math. J. 33 (1984), 51-87.

[4] M.F. Bidaut-Véron, Self-similar solutions of the p-Laplace heat equation: the fast diffusion case. Pacific J. Math.2, 227 (2006), 201-269.

[5] M.F. Bidaut-Véron, and S. Pohozaev. Nonexistence results and estimates for some nonlinear elliptic problems. J.Anal. Math 84 (2001) 1-49.

[6] Bouzelmate, A. Gmira, G. Reyes, Radial selfsimilar solutions of a nonlinear Ornstein-Uhlenbeck equation. Electronic Journal of Differential Equations, Vol.2007(2007), No.67, pp 1-21.

[7] H. Brezis, L.A. Peletier and D. Terman. A very singular solution of the heat equation with absorption, Arch. Rational Mech. Anal. 96 (1985), 185-209.

[8] L.A. Caffarelli, B. Gidas and J. Spruck. Asymptotic symmetry and local behavior of semilinear elliptic equations with critical Sobolev growth. Comm. In Pure and App Math. 42 (1989) 271297. 
[9] J.I. Diaz and J.E. Saa. Uniqueness of very singular self-similar solution of a quasilinear degenerate parabolic equation with absorption. Publ. Matem. 36 (1992), 19-38.

[10] R.H. Fowler, The form near infinity of real continuous solutions of a certain differential equation of second order. Quart. J1. Math 45 (1914) 289-350.

[11] R.H. Fowler. The solutions of Emden's and similar differential equation. Monthly notices Roy. Astr. Soc 91 (1920) 63-91.

[12] R.H. Fowler. Further studies on Emden's and similar differential equation. Quart. J1. Math 2 (1931) 259-288.

[13] B. Franchi, E. Lanconelli and J. Serrin. Existence and uniqueness of nonnegative solutions of quasilinear equations in $\mathbb{R}^{N}$. Advances in Math. 118 (1996).

[14] B.H. Gilding and L.A. Peletier. On a class of similarity solutions of the porous media equation. II, J. Math. Anal. Appl. 57 (1977), 522-538.

[15] B.H. Gilding and L.A. Peletier. On a class of similarity solutions of the porous media equation. J. Math. Anal. Appl. 55 (1976), 351-364.

[16] M. Guedda and L. Véron. Local and global properties of solutions of quasilinear equations. J1. Diff. Eq 76 (1988) N1, 159-189.

[17] M.A. Herrero and M. Pierre. The Cauchy problem for $u_{t}=\Delta u^{m}$ when $0<m<1$. Trans. Am. Math. Soc. 291 (1985), 145-158.

[18] S. Y. Hsu. Singular limit and exact decay rate of a nonlinear elliptic equation. Nonlinear Anal, 75 (7) (2012) 3443-3455.
[19] S. Y. Hsu. Exact decay rate of a nonlinear elliptic equation related to the Yamade flow. Proc. Amer. Math Soc, 142 (12) (2014) 4239-4249.

[20] K. M. Hui. Asymptotic behaviour of solutions of the fast diffusion equation near its extinction time. Math. Anal. Appl. 454 (2017) 695-715.

[21] G. Leoni. A very singular solution for the porous media equation $u_{t}=\Delta\left(u^{m}\right)-u^{p}$ when $0<m<$ 1. J. Differential Equations, 132, 353-376 (1996).

[22] G. Leoni. Classification of positive solutions of the problem div $\left(|\nabla u|^{p-2} \nabla u\right)+x . \nabla\left(u^{q}\right)+\alpha u^{q}=$ 0 in $\mathbb{R}^{N}$. Differential Equations, Vol 34, No9, (1998), 1172-1180.

[23] W.M. Ni and J. Serrin. Existence and nonexistence theorems for ground states of quasilinear partial differential equations. The anomalous case. Accad. Naz. Lincei, Convegni dei Lincei 77 (1986), 231-257.

[24] W. M.Ni and J. Serrin. Nonexistence theorems for singular solutions of quasilinear partial differential equations. Comm. Pure and Appl.Math 38 (1986) 379-399.

[25] J.L.Vazquez. Smoothing and decay estimates for nonlinear diffusion equations. Equations of porous medium type. Oxford Lecture Series in Mathematics and its Applications, vol. 33, Oxford University Press, Oxford, 2006.

[26] J.L.Vazquez. The porous medium equation. Mathematical theory . Oxford Mathematical Monographs, The Clarendon Press, Oxford University Press, Oxford, 2007.

\section{Creative Commons Attribution License 4.0 (Attribution 4.0 International, CC BY 4.0)}

This article is published under the terms of the Creative Commons Attribution License 4.0 https://creativecommons.org/licenses/by/4.0/deed.en US 